\title{
Subject Index Vol. 6,1996
}

\section{Verhaltenstherapie}

Action vs. state orientation 133 Adolescents 288 Aetiology 4210 Aggression 114 Agoraphobia 114 Anxiety 288

Atopic dermatitis, psychological factors 276

Behavior therapist (self-assessment;

assessment by psychoanalysists) 294 Behavioral medicine 122, 2 76, 4210 Biofeedback 143 Biographical analysis 4244

Change of paradigm 4244

Chronic pain 143

Clinical Psychology and behavior

therapy 3170 Cognitive behavior therapy 133 Consultation-liaisonservice 122

Depression 288

Diagnostics and intervention 3170 Differential diagnosis 2100 Dual diagnosis 4201

East-West differences 288 Ecstasy 4222 Effect sizes 14 Ego-dystonic 3162 Ego-systonic 3162 Erythrophobia 133 Evaluation 4201 
Follow-up 122

Gilles de la Tourette syndrome 2100 Group therapy 3143

Hallucinogenes 4222 Hypertension 143

'Ideot Savant' 4234 Image of behavior therapy 294 Imagery 3124 Imagery training 3124 Imaginative methods 3124 Imagination 3124 Incontinence 143 Individualization of therapeutic

strategies 4244 Inpatient treatment 4201

Mental retardation 4234

Meta-analysis 14

Migraine 4210

Models for disorder and treatment

4244

Neurophysiology 4210

Obsessive compulsive disorder (OCD)

3 143,3 162 Obsessive-compulsive personality disorder (OCPD) 3162

Panic disorder 114, 3135 Partner conflict 114 
Personality disorders 4201

Pilot project 4201

Plan analysis 133

Prevalence 4222

Psychosocial methods of intervention

14 Psychotherapist training, practical 2107 Public health 3170

Quality assurance 3170

Research and practice relationship 3170

Schizophrenia 14,4 201 Schizotypy 288

Scientist-practitioner model 2107 Self-efficacy 143 Self-Help groups 3143 Short-term behavior therapy 122 Somatoform disorders 122,143 Standardized therapy 4244 Stimulants 4222 Styles of Conflict Inventory 114 Substance abuse 4222 Substance disorders 4201 Supervision 2107

Therapy outcome studies 3135 Treatment fidelity 3135

Use of categorial systems 3135

Video feedback 3162

276

Hinweìse für Autoren

Verhaltenstherapie 1996;6:3. Umschlagseite

Ethische und rechtlíche Voraussetzungen: Zur Publikation eingereichte Manuskripte müssen bei Untersuchungen an Probanden oder Patienten die Erklärung enthalten, daß das VersuchsprotokoU von einer Ethikkommission genehmigt wurde und somit den ethischen Standards der Deklaration von Helsinki 1964 und ihren Ergänzungen entspricht. Gleichzeitig ist die Einwilli-gung der Versuchsperson nach Aufklärung im Text des Manuskriptes zu fixieren. Hinweise, die auf die Identität der Versuchsperson schließen lassen, sind zu vermeiden.

1. Allgemeines, Verlagsrecht

Bitte senden Sie Ihr Manuskript direkt an den Verlag S. Karger, z.Hd. Frau Susanne Meister, Lörracher Str. 16a, D-79115 Freiburg.

Beiträge erscheinen als Originalarbeiten, Kurzmitteilungen, Kasuistiken, Aus der Praxis für die Praxis, Diskussionsforum.

Das Manuskript soil in satzreifem Zustand in vierfacher Ausfertigung ein-gereicht werden, wahlweise auf Diskette gespeichert plus 3 fach-Ausdruck (bevorzugt Microsoft Word auf 3,5-Zoll-, MS-DOS-formatierten Disketten), zusammen mit reproduktionsfähigen Abbildungsvorlagen. Eine Kopie des Manuskriptes soil grundsätzlich beim Autor verbleiben. Die Entscheidung über die Annahme wissenschaftlicher Beiträge erfolgt aufgrund mehrerer Gutachten; alle veröffentlichten Arbeiten sind also «peer reviewed*. Typographische Gestaltung und redaktionelle Bearbei-tung sind dem Verlag vorbehalten. Für jedes Manuskript muß in einem Begleitschreiben von alien Autoren durch Unterschrift bestätigt werden, daß die eingereichte Arbeit oder Teile hiervon (Ausnahme: Kongreßberichte) bislang weder veröffentlicht wurde(n) noch gegenwärtig zur anderweitigen Veröffentlichung eingereicht ist/sind, sowie daß alle Autoren das Manuskript gelesen haben und mit der Veröffentlichung in der vorliegenden Form einverstanden sind. Es ist Sache des Autors, die

Nachdruckerlaubnis für Abbildungen, Tabellen usw. aus ande-ren Publikationen zu beschaffen. Mit der Annahme des Manuskriptes zur Publikation gehen alle Rechte auf den Verlag über. Ohne schriftliche Genehmigung des Verlages dürfen diese Publikationen oder Teile daraus nicht in andere Sprachen übersetzt oder in irgendeiner Form mit mechani-schen oder elektronischen Mitteln (einschließlich Fotokopie, Tonaufnahme und Mikrokopie) reproduziert oder auf einem Datenträger oder einem Computersystem gespeichert werden.

Geschützte Markennamen müssen mit einem hochgestellten ${ }^{\circledR}$ bezeichnet werden. Die Verantwortung für die korrekte Befolgung dieser Vorschrift liegt ausschließlich beim Autor.

2. Manuskripte

Originalarbeiten (Gliederung s.u.) sollen 10 Textseiten nicht überschreiten. Kurzmitteilungen folgen der Gliederung von Originalarbeiten und sollen 5

Textseiten, 5-10 Literaturhinweise und max. 1 Abbildung und 1 Tabelle (oder wahlweise 2 Abbildungen oder Tabellen) nicht überschreiten. Schwerpunkte von Kurzmitteilungen sollen neue Ergebnisse wissenschaftlicher Arbeiten, auch in Form sogenannter vorläufiger Mitteilungen, und auch neue Studienansätze experimentell oder klinisch - sein. Verlag und Schriftleitung wollen erreichen, daß solche Arbeiten innerhalb kürzester Zeit publiziert werden.

Selbstverständlich bleibt es dem Autor überlassen, zu einem späteren Zeitpunkt eine ausführliche Fassung einzureichen. Manuskripte sollen in 1¹/8zeiligem Abstand geschrieben sein, links und rechts einen angemessenen breiten Rand aufweisen und (einschließlich Titelseite und Literaturverzeichnis) kontinuierlich durchnumeriert sein. Für Umfangsüberschreitungen wird dem Autor ein Mehrkostenanteil in Rechnung gestellt (s.u.). Folgende Gliederung wird empfohlen: Titelseite mit Titel und Kurztitel der Arbeit in Deutsch und Englisch; Autorenangabe; Institutsangabe in der Landessprache der Autoren.

Zusammenfassung und Schlüsselwörter in Deutsch und Englisch. Zusam-menfassungen sollen 20 Schreibmaschinenzeilen nicht überschreiten. In die Zusammenfassung gehören keine Literaturzitate, Tabellen, Abbildungen und Abbildungs- und Tabellenhinweise sowie allgemein gehaltene Aus-sagen. Zu einer Arbeit gehören 3-5 Schlüsselwörter.

Der Textteil einer Arbeit soil gegliedert sein in Einleitung, Material und Methoden (oder: Patientengut und Methoden). Ergebnisse, Diskussionen.

Fußnoten (gegebenenfalls numeriert) am unteren Rand jeder Textseite. Abkürzungen bitte möglichst sparsam verwenden. Bei spezìellen Abkür-zungen jeweils beim ersten Auftreten das Wort ausschreiben und die später 
verwendete Abkürzung in Klammern folgen lassen: z. B. Adenosin-Mono-phosphat (AMP).

Danksagungen sollten unmittelbar dem Text folgen und dem Literaturverzeichnis vorangestellt sein.

Das Literaturverzeichnis soil nur die im Text zitierten Publikationen enthalten und in alphabetischer Reihenfolge nach Autorennamen geordnet sein. Im Literaturverzeichnis sollen sämtliche Autoren einer Arbeit notiert werden, die Abkürzung «et al.» ist zu vermeiden. Referenznummern werden nicht verwendet. Im Text werden bis zu zwei Autoren in eckigen Klammern erwähnt (ohne Initialen), bei drei und mehr Autoren ist nur der Name des ersten Autors, gefolgt von «et al.», zu notieren. Es sollen nur Arbeiten, die entweder publiziert oder zur Publikation angenommen sind, zitiert werden. Der Passus «in Vorbereitung» oder «persönliche Mitteilung» ist zu vermeiden.

Zur Abfassung von Literaturzitaten gelten folgende Beispiele:

Darstellungsschema einer Zeitschriftenreferenz:

Kauffman HF, van der Heide S, Beaumont F, Blok H, de Vries K: Class-specific antibody determination against Aspergi;lus fumigatus by means of the enzyme-linked immunosorbent assay. III. Comparative study: IgG, IgA, IgM ELISA titers, precipitating antibodies and IgE binding after fractiona-tion of the antigen. Int Arch Allergy Appl Immunol 1986;80:300-306.

Darstellungsschema einer Buchreferenz:

Hardy WD Jr, Essex M: FeLV-induced feline acquired immune deficiency syndrome: A model for human AIDS; in Klein E (ed): Acquired Immunodeficiency Syndrome. Prog Allergy. Basel, Karger, 1986, vol 37, pp 353-376.

Die Zahl von Abbildungen und Tabellen soil niedrig gehalten werden und darauf abzielen, den Text zu erläutern. Abbildungen werden mit (Abb. 1), Tabellen mit (Tab. 1) in den Text eingefügt. Bereits im Text genannte Zahlen bedürfen keiner Wiederholung in einer Tabelle. Umge-kehrt brauchen die in Tabellen zusammengestellten Zahlen nicht im Text wiederholt zu werden. Farbabbildungen können angenommen werden, bedürfen aber eines Reproduktìons- und Druckkostenzuschusses, den der Autor zu tragen hat (s.u.).

Abbildungsvorlagen sind 4fach als Papierabzüge einzureichen (Original + Kopien). Vorlagen nicht aufkleben, lochen oder mit Büroklammern an-heften; auf der Rückseite den Namen des Autors, den Titel des Beitrags, die Angabe, wo «oben» ist, sowie die Bildnummer angeben. Alle Abbildungen und Tabellen sind mit einer Legende zu versehen. Alle Legenden einer Arbeit werden auf einer gesonderten Seite zusammenge-stellt. Die Legenden sind kurz und präzise zu halten und sollen die Abbildungen bzw. Tabellen vollständig erklären. Die Interpretation der Daten erfolgt nicht in der Legende, sondern ausschließlich im Text.

Autorenadresse

Postanschrift des Autors am Schluß der Arbeit vollständig angeben.

3. Kosten und Sonderdrucke

Übersteigen die Kosten der Autorkorrektur 10\% der Satzkosten, werden diese dem Autor in Rechnung gestellt.

Sollte eine Arbeit den vorgegebenen Umfang überschreiten und vom Autor nicht gekürzt werden, wird für den Mehrumfang pro Druckseite der Betrag von DM 650.- in Rechnung gestellt.

Für Farbabbildungen wird dem Autor ein Reproduktìons- und Druckkostenanteil von DM 450,- pro Abbildung in Rechnung gestellt. Dem mit Postanschrift genannten Autor stehen 25 kostenlose Sonderdrucke seiner Arbeit zu.

\section{KARGER}


(C) 1996 S. Karger GmbH, Freiburg

Fax (07 61) 4520714

http://www.karger.ch/journals/ver/verdes.htm 